\title{
Altered levels of circulating insulin-like growth factor I (IGF-I) following ischemic stroke are associated with outcome - a prospective observational study
}

N. David Åberg ${ }^{1 *}$, Daniel Åberg ${ }^{1}$, Katarina Jood ${ }^{2}$, Michael Nilsson ${ }^{2,3}$, Christian Blomstrand ${ }^{2}$, H. Georg Kuhn $n^{2,4}$, Johan Svensson ${ }^{1}$, Christina Jern ${ }^{5,6}$ and Jörgen Isgaard ${ }^{1}$

\begin{abstract}
Background: Insulin-like growth factor I (IGF-I) has neuroprotective effects in experimental ischemic stroke (IS). However, in patients who have suffered IS, various associations between the levels of serum IGF-I (s-IGF-I) and clinical outcome have been reported, probably reflecting differences in sampling time-points and follow-up periods. Since changes in the levels of post-stroke s-IGF-I have not been extensively explored, we investigated whether decreases in the levels of s-IGF-I between the acute time-point (median, 4 days) and 3 months ( $\Delta \mid$ GF-I, further transformed into $\Delta \mid$ GF-I-quintiles, $\Delta \mathrm{GF}-\mathrm{I}-\mathrm{q})$ are associated with IS severity and outcome.

Methods: In the Sahlgrenska Academy Study on Ischemic Stroke (SAHLSIS) conducted in Gothenburg, Sweden, patients with IS who had s-IGF-I measurements available were included ( $N=354 ; 65 \%$ males; mean age, 55 years). Baseline stroke severity was evaluated using the National Institutes of Health Stroke Scale (NIHSS) and converted into NIHSS-quintiles (NIHSS-q). Outcomes were assessed using the modified Rankin Scale (mRS) at 3 months and 2 years.

Results: In general, the levels of s-IGF-I decreased (positive $\Delta \mid G F-I)$, except for those patients with the most severe NIHSS-q. After correction for sex and age, the 3rd $\Delta$ IGF-I-q showed the strongest association to mRS 0-2 [Odds Ratio (OR) 5.11, 95\% confidence interval (CI) 2.18-11.9], and after 2 years, the 5th $\Delta$ IGF-I-q (OR 3.63, 95\% Cl 1.40-9.38) showed the strongest association to mRS $0-2$. The associations remained significant after multivariate correction for diabetes, smoking, hypertension, and hyperlipidemia after 3 months, but were not significant $(p=0.057)$ after 2 years. The 3-month associations withstood additional correction for baseline stroke severity $(p=0.035)$, whereas the 2-year associations were further attenuated $(p=0.31)$.

Conclusions: Changes in the levels of s-IGF-I are associated primarily with temporally near 3-month outcomes, while associations with long-term 2-year outcomes are weakened and attenuated by other factors. The significance of the change in post-stroke s-IGF-I is compatible with a positive role for IGF-I in IS recovery. However, the exact mechanisms are unknown and probably reflects combinations of multiple peripheral and central actions.
\end{abstract}

Keywords: Insulin-like growth factor I, Ischemic stroke, Outcome

\footnotetext{
* Correspondence: david.aberg@medic.gu.se

${ }^{1}$ Department of Internal Medicine, Institute of Medicine, The Sahlgrenska

Academy at University of Gothenburg, Gröna Stråket 8, SE-413 45

Gothenburg, Sweden
}

(c) The Author(s). 2018 Open Access This article is distributed under the terms of the Creative Commons Attribution 4.0 International License (http://creativecommons.org/licenses/by/4.0/), which permits unrestricted use, distribution, and reproduction in any medium, provided you give appropriate credit to the original author(s) and the source, provide a link to the Creative Commons license, and indicate if changes were made. The Creative Commons Public Domain Dedication waiver (http://creativecommons.org/publicdomain/zero/1.0/) applies to the data made available in this article, unless otherwise stated. 


\section{Background}

Extensive studies conducted in experimental animals have demonstrated that insulin-like growth factor-I (IGF-I) exerts neuroprotective and plasticity-promoting effects $[1,2]$. For humans who have suffered an ischemic stroke (IS), a few observational studies have evaluated the role of endogenous levels of serum IGF-I (s-IGF-I). In the first two studies on this topic ( $N=85$ and $N=42$, respectively), s-IGF-I was associated with measures of improved functional outcome [3, 4]. However, s-IGF-I was analyzed at only one time-point, either within $24 \mathrm{~h}$ of IS onset [3] or at 19-209 days after the IS [4], and functional follow-up was performed approximately $3-6$ months post-stroke $[3,4]$. In addition, a previous study from our group $(N=407)$ has shown a positive association between the 3-month level of s-IGF-I and improvements in the mRS score from 3 months to 2 years, whereas there was a negative association with the 3-month mRS score [5]. Thus, although endogenous s-IGF-I has been associated with favorable IS outcome, there remain unresolved issues in terms of the importance of the: post-stroke sampling time-point; age of the patient; severity of IS; timing of the follow-up; and temporal changes in s-IGF-I level after IS.

The association between change in post-stroke s-IGF-I and IS outcome has been investigated in one small study, in which the average level of s-IGF-I increased by $8.5 \%$ from $<72$ h to 7 days after the stroke $(N=15)$ [6]. However, in that study, there was relatively large inter-individual variability, and interestingly, a decrease in post-stroke s-IGF-I correlated with a better1-month mRS score $(N=10)$ [6]. Furthermore, the local expression of IGF-I in the brain may increase after IS [7, 8], and in analogy with the increased brain uptake of IGF-I after experimental exercise [9], the transport of IGF-I from the serum into the brain may be increased after IS. In our previous study, the level of s-IGF-I was increased during the first days after the stroke $(+11.2 \%$ on Days $0-2$, as compared to healthy controls), followed by a leveling off of the s-IGF-I level on Days 3-5. Thereafter, there was an average decrease of $14.8 \%$ from Day 9 to Day 19, resulting in 3-month s-IGF-I levels that were approximately similar to those seen in healthy, age-matched controls [5]. However, we did not analyze the associations between the individual changes in s-IGF-I and IS outcome [5]. The hypothesis underlying the present study is that not only the absolute levels of s-IGF-I, but also the temporal pattern of s-IGF-I levels (as estimated by changes in the s-IGF-I levels), are of importance for IS outcome. Thus, our primary objective was to investigate whether intra-individual changes in post-stroke s-IGF-I ( $\Delta \mathrm{IGF}-\mathrm{I})$, from the acute phase to 3 months post-IS, are associated with functional independence 3 months after the IS, and if so, whether $\triangle I G F-I$ is also associated with functional outcome 2 years after IS. As $\triangle I G F-I$ has not been investigated extensively to date, we explored descriptively the effects of the following parameters: first day of sampling; age; IS severity; stroke subtype; and stroke etiology. We also performed multivariate regression analyses with the inclusion of potential confounders, such as cardiovascular risk factors and IS severity.

\section{Methods}

\section{Subjects and methods}

The design of SAHLSIS has been reported elsewhere [10]. Briefly, patients ( $<70$ years of age) with first-ever or recurrent acute IS were recruited consecutively at four Stroke Units in western Sweden between 1998 and 2003 (see Fig. 1 for flow chart of inclusions). The final inclusion cohort with regard to $\triangle$ IGF-I had 354 subjects (Table 1). S-IGF-I was analyzed on one occasion in 2008 with a methodological intra-assay coefficient of variation (CV) of 5.1\% and the biological variation showed a CV of 38\% [5]. Blood sampling was performed between 08:30 and 10:30 after overnight fasting, and s-IGF-I was assayed using an IGF-binding protein (IGFBP)-blocked RIA kit (Mediagnost, Reutlingen, Germany). The acute serum samples were taken 0-19 days after the IS, with a median sampling time of 4 days [5]. The frequencies of previous hypertension, diabetes mellitus, and smoking were recorded and, the levels of low-density lipoprotein (LDL) were evaluated as previously described [10]. The $\triangle \mathrm{IGF}-\mathrm{I}$ values were transformed into quintiles $(\Delta \mathrm{IGF}-\mathrm{I}-\mathrm{q})$, owing to the values being skewed towards the left, as well as for convenience of presentation. The $\Delta$ IGF-I-q were defined according to $\triangle$ IGF-I: q1 = $-279.6--10, \quad \mathrm{q} 2=-9.999-10, \quad \mathrm{q} 3=10.001-30.7, \quad \mathrm{q} 4=$ $30.701-55.6$, q5 $=55.601-178.9 \mathrm{ng} / \mathrm{ml}$, with higher quintiles representing a decrease in s-IGF-I from the acute time-point to the 3-month time-point. Blood glucose or plasma glucose was analyzed using standardized methods at the Department of Clinical Chemistry at the Sahlgrenska University Hospital. In cases with the presence of blood glucose, these values were transformed to plasma glucose according to the formula: plasma glucose $=$ blood glucose $\times 1.11$. Initial stroke severity was assessed by scoring using the Scandinavian Stroke Scale (SSS), with the values being recalculated into the now more commonly used National Institutes of Health Stroke Scale (NIHSS). The algorithm used was: NIHSS $=25.68-0.43 \times$ SSS [11] , and due to a markedly skewed appearance, these scores were further transformed into quintiles: $\mathrm{q} 1=0-0.74$ (mild); $\mathrm{q} 2=$ 0.7401-2.03 (minor); q3 = 2.0301-3.75 (moderate); q4 = 3.75-10.2 (major); and q5 =10.201-42 (severe). Due to many cases having minimal NIHSS scores, the first quintile was somewhat overbalanced (see Fig. 2). Stroke subtype and etiology were classified according to the Oxfordshire Community Stroke Project (OCSP) [12] and the Trial of Org 10,172 in Acute Stroke Treatment (TOAST) [13] criteria (see Table 2 for groups and abbreviations). 


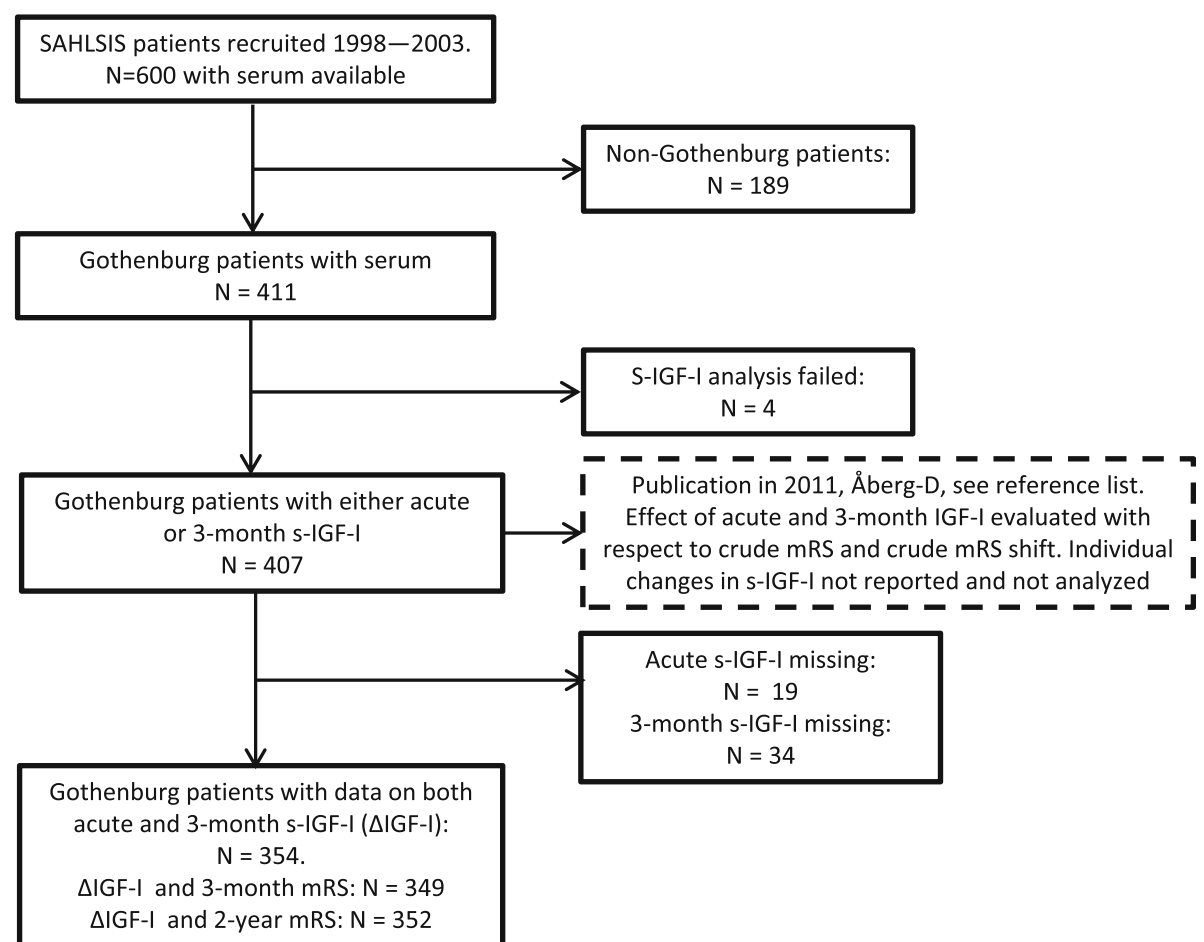

Fig. 1 Flow chart showing numbers of included subjects and reasons for the exclusion of other patients

Outcomes were assessed functionally and classified according to the modified Rankin Scale (mRS), where the levels of functional independence was classified by scores in the range of $0-2$ (considered as favorable) and scores in the range of 3-6 (considered as unfavorable) [14]. Further details of the study design, patient examination, scoring scales, and protein measurements are given in the Additional file 1. A part of this report was presented as an abstract (with a modified title) at the European Stroke Conference in May 2018 [15] .

\section{Statistical analysis}

Statistical evaluation was performed using the SPSS ver. 21.0 software (SPSS Inc., Chicago, IL). In the descriptive section, comparisons between groups (stroke severity, day-of-sampling, age, stroke subtype, and etiology) were performed using analysis of variance (ANOVA), and with Dunnett's (for comparison with one reference) or Tukey's (for crosswise comparison of all groups) post hoc tests, as indicated. Comparisons between distributions were made with the Chi-square test. Crude correlations using the method of Pearson are presented.

The aim of the project was to evaluate the effect of $\triangle$ IGF-I on functional outcome (mRS) 3 months and 2 years after IS. Towards this goal, we assessed whether the crude correlations withstood subsequent binary logistic regression analysis, in which the data on functional outcome were dichotomized (favorable outcome, mRS
$0-2$ versus unfavorable outcome, mRS 3-6). The Odds Ratios (ORs) and 95\% confidence intervals (CIs) for favorable outcome (mRS 0-2) were age- and sex-adjusted (model 1) and were relative to the lowest quintile of $\triangle I G F-I$ ( $\triangle I G F-I-q 1$, i.e., an increase in $\triangle \mathrm{IGF}-\mathrm{I})$. Adjustments were also made for the vascular risk factors of smoking, hypertension, diabetes, and LDL levels (model 2), initial stroke severity quintile (model 3), and finally, also for the different days of first sample (model 4). As suggested by Peduzzi and coworkers [16], the number of events per variable (EPV) needed to reduce statistical bias should exceed the included number of covariates by a factor of 10 . With the eight covariates given above, and an event rate of $\mathrm{mRS}$ 3-6 $(N=82$ and $N=74$ for the 3-month and 2-year outcomes, respectively), we refrained from adding 'stroke etiology' and 'stroke subtype' as covariates. The statistical significance level was set at $p<0.05$.

\section{Results}

Descriptive data for s-IGF-I and stroke severity and subtype The baseline characteristics of the 354 patients from SAHLSIS (Fig. 1) with $\triangle$ IGF-I values are summarized in Table 1. $\triangle$ IGF-I, which represents the intra-individual decrease in s-IGF-I from the acute phase to 3 months post-IS, averaged $20.2 \mathrm{ng} / \mathrm{mL}$ for the entire group. $\triangle \mathrm{IGF}-\mathrm{I}$ only weakly correlated with age $(r=-0.12, p=0.025, N=$ $354)$, as compared to the acute s-IGF-I and age $(r=-0.331$, $p<0.001, N=354)$ and the 3-month s-IGF-I and age 
Table 1 Baseline data for patients and s-IGF-I in each of the quintiles of changing s-IGF-I ( $\Delta$ IGF-I-q1-5)

\begin{tabular}{|c|c|c|c|c|}
\hline \multicolumn{2}{|l|}{ Parameter } & \multicolumn{2}{|l|}{ Unit } & Value \\
\hline \multicolumn{4}{|l|}{$n$} & 354 \\
\hline \multicolumn{2}{|l|}{ Age at index ischemic stroke } & \multicolumn{2}{|l|}{ Years (SD) } & $55.4(11)$ \\
\hline \multirow{2}{*}{\multicolumn{2}{|c|}{ Sex }} & \multicolumn{2}{|l|}{ Missing (N) } & 229/125 \\
\hline & & \multicolumn{2}{|c|}{ Male/female (fraction) } & 0.65 \\
\hline \multirow{2}{*}{\multicolumn{2}{|c|}{ Diabetes }} & \multicolumn{2}{|l|}{ Yes (N/fraction) } & $67(0.19)$ \\
\hline & & \multicolumn{2}{|l|}{ Missing (N) } & 0 \\
\hline \multirow{2}{*}{\multicolumn{2}{|c|}{ Hypertension }} & \multicolumn{2}{|l|}{ Yes (N/fraction) } & $188(0.53)$ \\
\hline & & \multicolumn{2}{|l|}{ Missing (N) } & 0 \\
\hline \multirow{2}{*}{\multicolumn{2}{|c|}{ Current smoking }} & \multicolumn{2}{|l|}{ Yes (N/fraction) } & $136(0.38)$ \\
\hline & & \multicolumn{2}{|l|}{ Missing (N) } & 0 \\
\hline \multirow{2}{*}{\multicolumn{2}{|c|}{ LDL level (ng/nL) }} & \multicolumn{2}{|l|}{ Mean (SD) } & $3.3(1.0)$ \\
\hline & & \multicolumn{2}{|l|}{ Missing (N) } & 27 \\
\hline \multirow{2}{*}{\multicolumn{2}{|c|}{ P-glucose (acute) }} & \multicolumn{2}{|l|}{ Mean (SD) } & $6.5(2.64)$ \\
\hline & & \multicolumn{2}{|l|}{ Missing (N) } & 8 \\
\hline \multirow{2}{*}{\multicolumn{2}{|c|}{ P-glucose (3 m) }} & \multicolumn{2}{|l|}{ Mean (SD) } & $6.03(2.29)$ \\
\hline & & \multicolumn{2}{|l|}{ Missing (N) } & 8 \\
\hline Stroke severity (NIHSS) & & Mean $(20,80 \%)$ & & $5.3(0.7,10.2)$ \\
\hline & & Missing (N) & & 0 \\
\hline Stroke outcome (mRS) $3 \mathrm{~m}$ & & Mean(SD) & & $1.85(1.06)$ \\
\hline & & Missing (N) & & 5 \\
\hline Stroke outcome (mRS) $2 \mathrm{yr}$ & & mRS (SD) & & $1.77(1.32)$ \\
\hline & & Missing (N) & & 2 \\
\hline Dead (3-24 m) & & Yes (n/fraction) & & $9(0.025)$ \\
\hline & & Missing (N) & & 0 \\
\hline s-IGF-I (acute) & & $\mathrm{ng} / \mathrm{mL}$ (SD) & & $172.8(62.9)$ \\
\hline & & Missing (N) & & 0 \\
\hline s-IGF-I (3 m) & & $\mathrm{ng} / \mathrm{mL}(\mathrm{SD})$ & & $152.7(55.7)$ \\
\hline & & Missing (N) & & 0 \\
\hline$\Delta \mathrm{IGF}-\mathrm{I}(\mathrm{ng} / \mathrm{mL})$, all data & & $\mathrm{ng} / \mathrm{mL}$ (SD) & & $20.2(51.0)$ \\
\hline & & Missing (N) & & 0 \\
\hline Quintile of $\Delta \mid G F-1$ : & & $\Delta \mathrm{IGF-I}(\mathrm{ng} / \mathrm{mL})$ & s-IGF-I acute (ng/mL) & Change (\%) \\
\hline & $\Delta|G F-|-q 1(S D)$ & $-48.3(48.0)$ & $146.5(53.7)$ & incr. 33.0 \\
\hline & $(\mathrm{N})$ & 71 & 71 & 71 \\
\hline & $\Delta|G F-|-q 2(S D)$ & $1.6(5.8)$ & $145.4(44.6)$ & decr. 1.10 \\
\hline & $(\mathrm{N})$ & 72 & 72 & 72 \\
\hline & $\Delta|G F-|-q 3(S D)$ & $20.8(5.7)$ & $152.5(56.0)$ & decr 13.5 \\
\hline & $(\mathrm{N})$ & 71 & 71 & 71 \\
\hline & $\Delta|G F-|-q 4(S D)$ & $42.5(7.2)$ & $178.9(45.9)$ & decr 23.6 \\
\hline & $(\mathrm{N})$ & 70 & 70 & 70 \\
\hline & $\Delta|G F-|-q 5(S D)$ & $85.9(28.0)$ & $240.5(57.6)$ & decr 35.7 \\
\hline & $(\mathrm{N})$ & 70 & 70 & 70 \\
\hline
\end{tabular}

Absolute $\Delta \mathrm{IGF}-\mathrm{I}(\mathrm{ng} / \mathrm{mL})$ represents a subtraction of acute s-IGF-I by 3-month s-IGF-I. A negative numerical value represents an increase from the acute to 3-month time point, and a positive numerical value represents a decrease. Modified Rankin scale (mRS), low density lipoprotein (LDL), National Institutes of Health Stroke Scale (NIHSS). An extended version of the table with a comparison to data presented in 2011 is found in Additional file 2: Table S1 


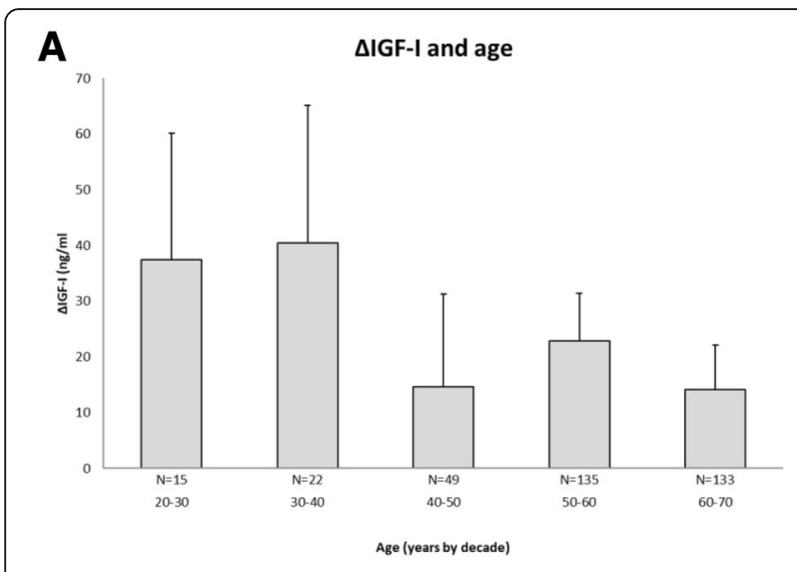

B $\Delta \mathrm{GGF-I}$ in different days after stroke

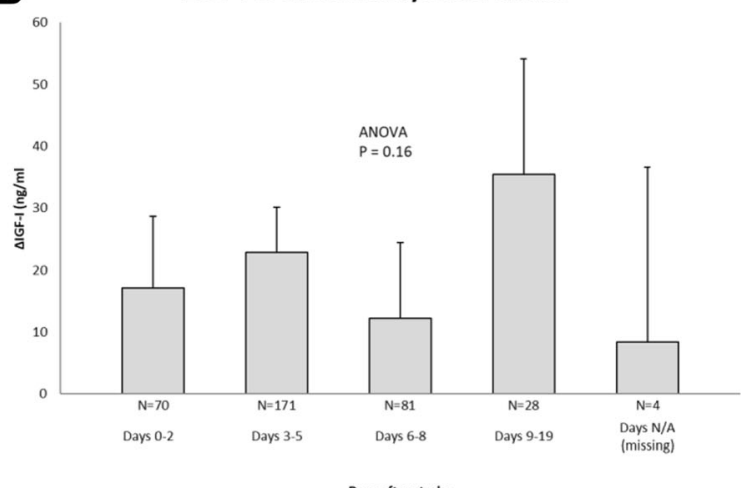

C $\quad \Delta \mathrm{IGF}-\mathrm{I}$ in different stroke severities

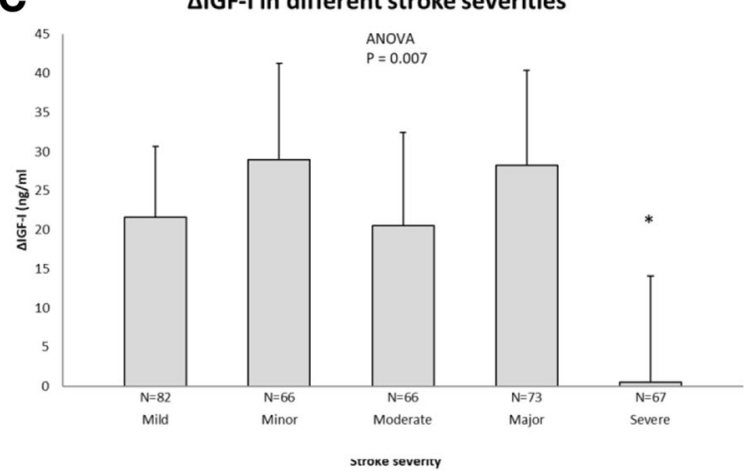

Fig. 2 Descriptive data on $\Delta \mid G F-1$ in relation to age, sampling day, and ischemic stroke (IS) severity. The error bars are 95\% confidence intervals $(\mathrm{Cl})$, and the numbers $(\mathrm{N})$ of subjects in each category are shown. a $\Delta \mid G F-I$ for patients of different ages, as expressed by age decade at index IS. b $\Delta$ IGF-I values for different days post-IS. c $\Delta$ IGF-I values for the five IS severity levels of the National Institutes of Health Stroke Scale (NIHSS) (for limits and details, see Methods section). Significance levels were analyzed by ANOVA, followed by post-hoc Dunnett's test with the first group as reference. If the ANOVA was non-significant no further testing was performed. ${ }^{*} p<0.05$

$(r=-0.264, p<0.001, N=354)$. Furthermore, the weak negative correlation between $\triangle \mathrm{IGF}-\mathrm{I}$ and age was not reflected in any difference of $\Delta \mathrm{IGF}-\mathrm{I}$ with respect to decade of age (Fig. 2a). We found no significant difference in
$\Delta$ IGF-I regardless of the post-stroke sampling day of the first "acute" serum sample (Fig. 2b). However, $\Delta$ IGF-I was found to be related to initial stroke severity (Table 2, Fig. 2c). Specifically, there was no decrease in $\triangle I G F-I$ in the most severe IS, whereas the value of $\triangle$ IGF-I (20$29 \mathrm{ng} / \mathrm{mL}$ ) was similar for all the other severities of IS. The low $\triangle$ IGF-I values noted for the patients with severe or large IS were further supported by the observed tendency towards a correlation between the $\triangle \mathrm{IGF}-\mathrm{I}$ quintiles and NIHSS quintiles $(r=-0.092, p=0.084, N=354)$, and the fact that the subtype with the largest IS, total anterior cerebral infarctions (TACI), had a lower $\triangle$ IGF-I than the other subtypes (Table 2, OCSP). Moreover, the $\triangle \mathrm{IGF}-\mathrm{I}$ value was higher in the group of patients with IS with etiology of arterial dissection, which might be attributable in part to the younger age of these patients (Table 2, TOAST classification). These subgroups were not used in the subsequent regressions, since the numbers of subjects and events in each of the groups were relatively low. As the absolute levels of s-IGF-I are inversely related to P-glucose levels and metabolic syndrome [17-19], $\Delta$ IGF-I could potentially relate to the P-glucose levels. However, there were no correlations (acute P-glucose and $\Delta \mathrm{IGF}-\mathrm{I} ; r=-0.065, p=0.23$, $N=346$; 3-month P-glucose and $\Delta$ IGF-I, $r=-0.012, p=$ $0.83, N=346$ ), and this parameter was not used in the subsequent regression analyses.

\section{Multivariate regression analysis of changes in the levels of s-IGF-I and the clinical outcomes 3 months and 2 years after the IS}

We investigated whether $\triangle \mathrm{IGF}-\mathrm{I}$ was related to IS outcome after 3 months and 2 years, using univariate (correlations) and multivariate regression analyses. Although $\triangle$ IGF-I correlated with the crude 3-month mRS score (Fig. 3a), outliers and the skewing of $\triangle$ IGF-I indicated that the $\triangle$ IGF-I quintiles were better suited to analysis of association, and the application of the widely accepted dichotomized mRS. Accordingly, the crude $\triangle I G F-I$ quintiles correlated with favorable outcome (i.e., mRS 0-2) 3 months after IS $(r=0.21, p=0.01, N=349)$ and 2 years after IS $(r=0.14, p=0.007, N=352)$. This is evidenced by the significantly different distributions of $\triangle \mathrm{IGF}-\mathrm{I}$ quintiles at both 3 months and 2 years for the patients with favorable outcome (mRS 0-2) and those with unfavorable outcome (mRS 3-6) of IS (Fig. 2b).

For the significant crude associations with mRS outcome 3 months and 2 years after the IS, we performed further analyses using binary logistic regression with adjustment for multiple covariates. Higher $\triangle I G F-I$ quintiles (representing decreased levels of s-IGF-I) adjusted for sex and age were indeed associated with favorable outcome in terms of mRS score after 3 months (model 1, for $\triangle$ IGF-I-q5, OR 4.63, 95\% CI 2.01-10.7; Fig. 3c). This was not due to the higher level of acute s-IGF-I found in 


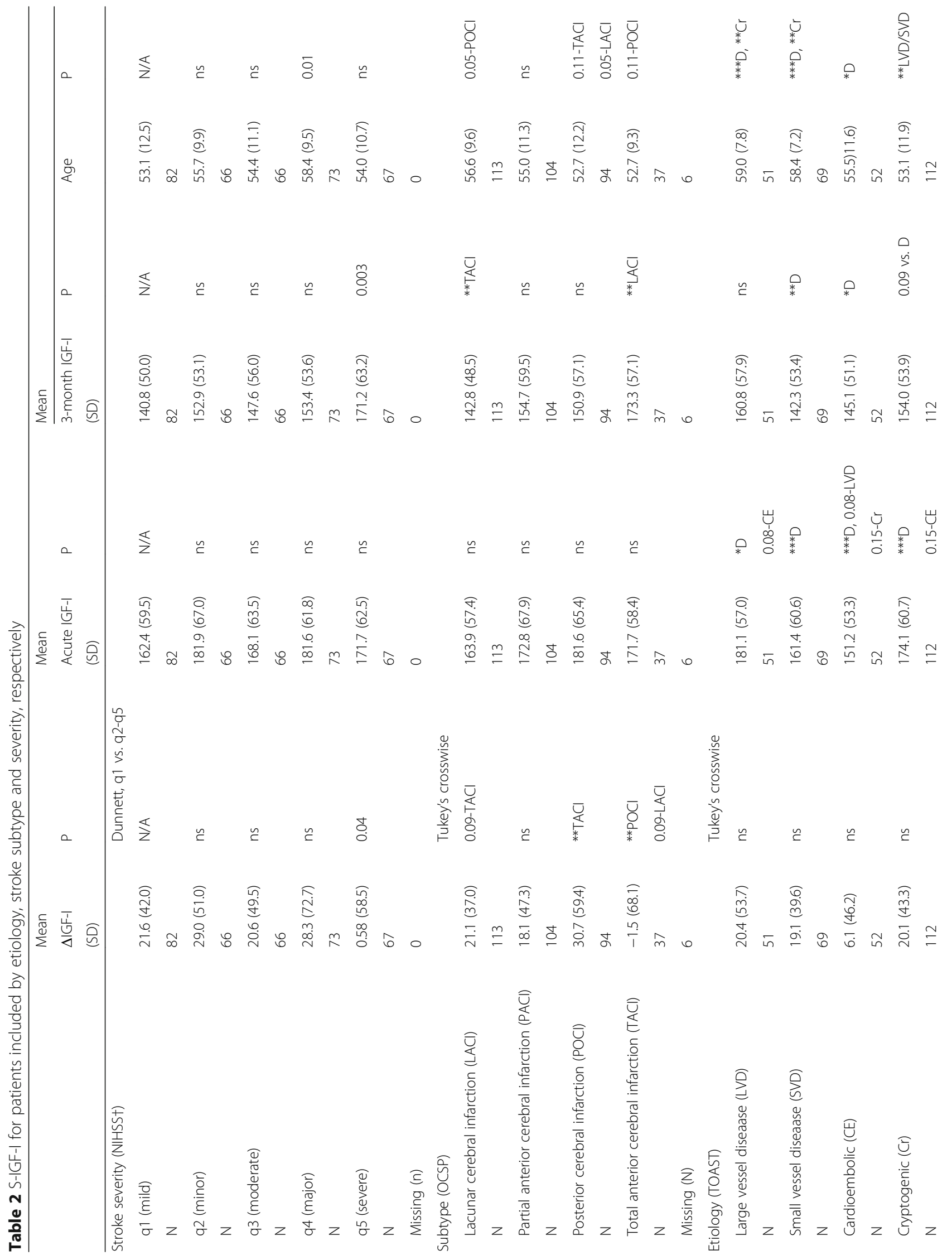


Åberg et al. BMC Neurology (2018) 18:106

Page 7 of 12

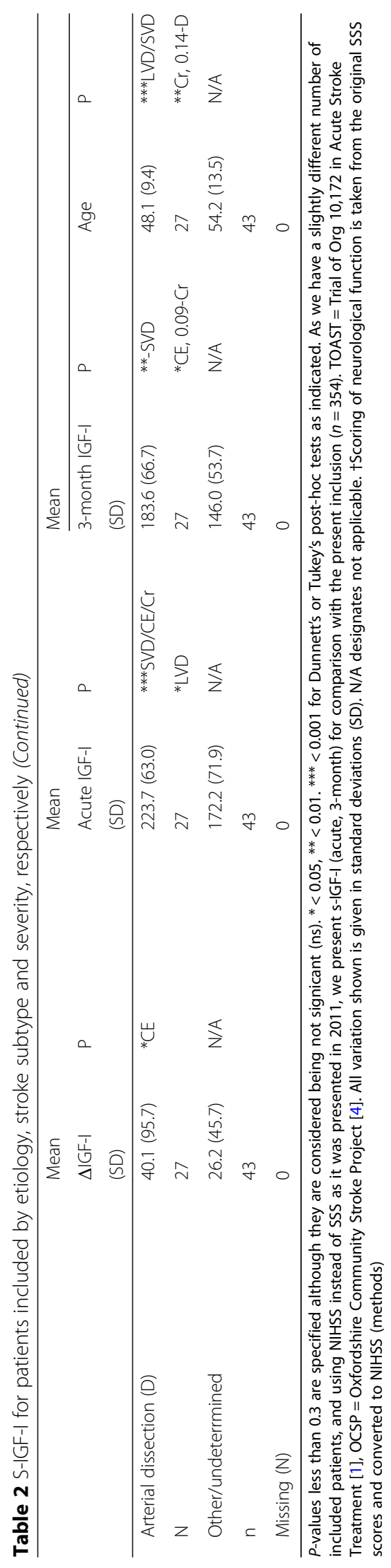




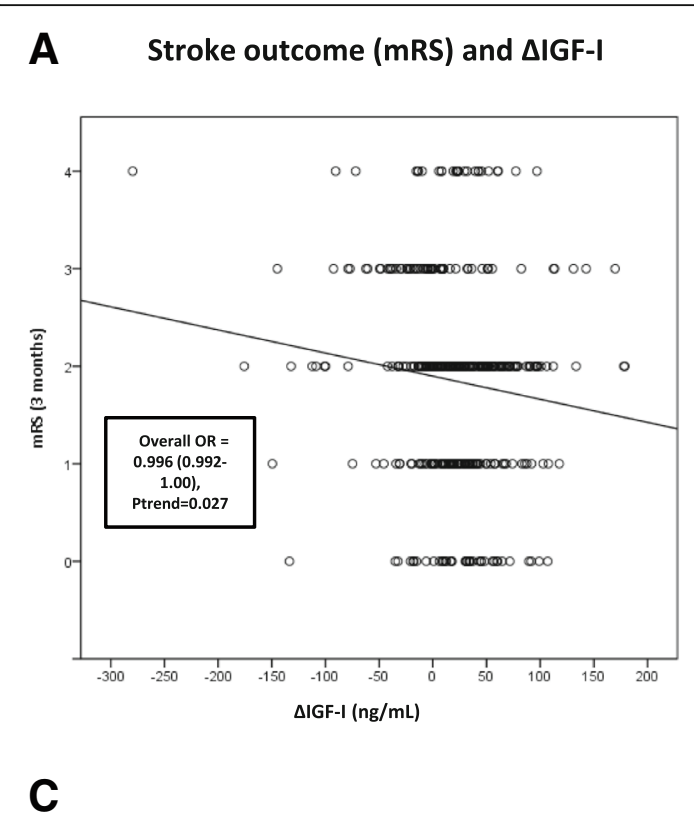

Stroke outcome 3 months (mRS 0-2)

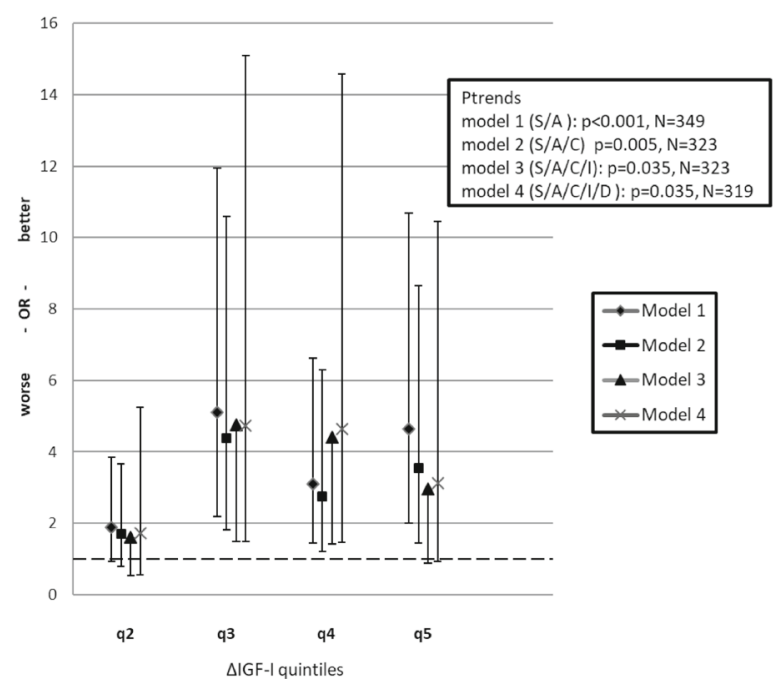

\section{B Stroke outcome and $\Delta$ IGF-I quintiles \\ $\mathrm{P}<0.001$ $P=0.024$}

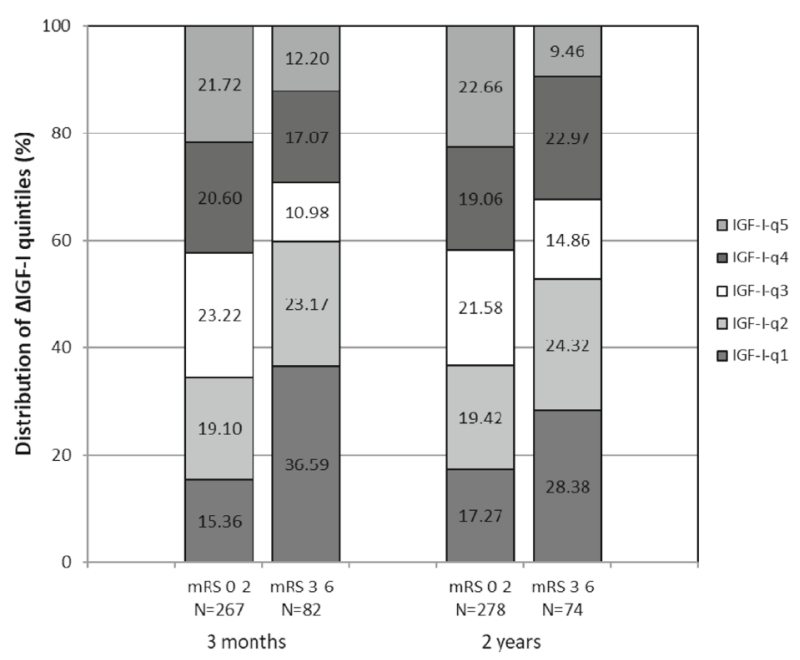

D

\section{Stroke outcome 2 years (mRS 0-2)}

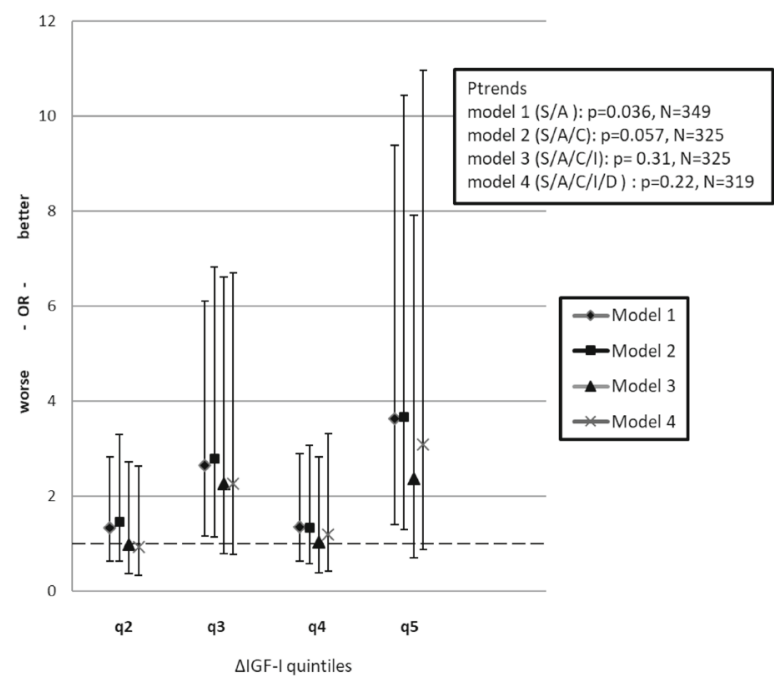

Fig. 3 Stroke outcome in relation to $\Delta \mid G F-I$. a. Distribution of crude $\Delta \mid G F-I$ values and crude 3-month mRS scores $(N=349)$. The box shows the overall Odds Ratios (OR) and 95\% confidence intervals of an ordinal regression with mRS score as the dependent variable. For convenience, the line represents a crude correlation $(r=-0.114, p=0.033)$. b. Unadjusted $\Delta \mid$ GF-I-quintile distribution (\%) of stroke outcomes as indicated by mRS scores of 0-2 (good or favorable) or 3-6 (poor or unfavorable) 3 months and 2 years after IS. The $p$-values from the Chi-square analysis comparing distributions of good and poor outcome are shown. c. Functional outcome 3 months post-IS, shown as OR and 95\% Cl for associations (binary logistic regression) of favorable $\mathrm{mRS}$ score with unfavorable functional outcome for each of the $\Delta \mid G F-I$ quintiles relative to $\Delta \mid G F-I$ q1 (q1 is a reference with $\mathrm{OR}=1$, shown as a hatched line). Models $1-4$ are shown with successively added adjustments for sex (S), age (A), traditional cardiovascular covariates (C), initial stroke severity (I), and day of the first blood sample (D), together with their respective numbers (N) with complete datasets. The boxes show the p-values for the overall associations using $\Delta \mid G F-I$ quintiles as a continuous variable with the same respective adjustments ( $p$-trends). $\mathbf{d}$. Functional outcomes 2 years after IS shown as OR and 95\% Cl for associations (binary logistic regression) between favorable mRS score and unfavorable functional outcome for each of the $\Delta|G F-|$ quintiles, as in B

DIGF-I-q4-5 (model 1 with acute s-IGF-I as an additional covariate; data not shown). We did not explore the impact of acute s-IGF-I on outcome any further, as acute s-IGF-I has been previously demonstrated to be negatively correlated with outcome [5]. Furthermore, the 3-month associations withstood adjustment for traditional cardiovascular risk factors and adjustment for initial stroke severity (Fig. 3c, model 3). However, the associations were marginally more robust for $\triangle \mathrm{IGF}-\mathrm{I}-\mathrm{q} 3$ than for $\Delta$ IGF-I-q5 (model 1, $\Delta$ IGF-I-q3: OR 5.11, 95\% CI 
2.18-11.9). Although 'day of first sample' did not crudely relate with $\triangle \mathrm{IGF}-\mathrm{I}$ (Fig. $3 \mathrm{~b}$ ), there was a biological rationale for including this parameter as a covariate (due to the initial increase in s-IGF-I; see Introduction), which marginally amplified the associations (Fig. 3, c and d, model 4). This was also reflected in the regression analysis in which samples collected on Days $0-2(N=70)$ were excluded, generating an inclusion cohort of $N=275$. This regression analysis generated marginally higher ORs for favorable outcome $(N=275$, model 1, $\Delta$ IGF-I-q3: OR 5.59, 95\% CI 2.03-14.4).

In general, the associations were somewhat weaker for the 2-year outcome (model 1, $\Delta$ IGF-I-q5: OR 3.63, 95\% CI 1.40-9.38). The association with favorable 2 -year outcome persisted as a trend $(p=0.057)$ after adjustment for cardiovascular risk factors but was significant for specific $\Delta$ IGF-I-quintiles (model 2, $\Delta$ IGF-I-q5: OR 3.66, 95\% CI 1.29-10.4). However, additional adjustment for initial stroke severity reduced associations to non-significant levels $(p=0.31)$. For the 2 -year outcome, the association was most robust for $\triangle \mathrm{IGF}-\mathrm{I}-\mathrm{q} 5$.

\section{Discussion}

\section{Decreased level of IGF-I is associated with favorable \\ outcome after ischemic stroke}

This study investigated individual s-IGF-I changes ( $\Delta \mathrm{IGF}-\mathrm{I})$ from the subacute phase after IS to 3-month follow-up. In addition, we related the $\Delta$ IGF-I to outcome for up to 2 years after IS. The $\Delta$ IGF-I did not differ with respect to stroke severity, except that in the most severe stroke cases, there was only a minimal change in the levels of s-IGF-I. In general, the individual $\triangle \mathrm{IGF}-\mathrm{I}$ values showed that s-IGF-I decreased from the subacute phase to 3 months post-IS. There were more prominent decreases in the levels of s-IGF-I in patients with favorable outcome than in those with unfavorable outcome. A subsequent regression analysis of $\triangle$ IGF-I-q revealed robust associations with favorable outcome at 3 months and somewhat less-pronounced associations at 2 years after IS. These associations withstood adjustments for cardiovascular covariates in the 3-month and 2-year follow-ups. However, the associations withstood additional adjustment for initial stroke severity only in terms of the 3-month follow-up. Taken together, our data show that a dynamic decrease in the level of s-IGF-I from the subacute phase to 3 months post-stroke is strongly associated with better stroke outcome at 3 months, whereas the association with outcome at 2 years is weaker.

\section{Decreasing the level of IGF-I is associated with favorable outcome, also after correction for confounders}

The effect of the decrease in the level of IGF-I might be confounded by various factors, such as stroke volume, initial stroke severity, higher initial s-IGF-I, and cardiovascular factors. While the exact stroke volume was not available, the TOAST classification gave some indication of the stroke volume [13]. Accordingly, large IS (TACI) and severe IS, as opposed to other groups, exhibited a minimal $\triangle I G F-I$ (i.e., unchanged s-IGF-I). Therefore, it could be argued that the worst IS scenario, with low $\triangle \mathrm{IGF}-\mathrm{I}$, could explain the statistical associations. However, even within the group of most severe IS, similar ORs for favorable outcome were noted (data not shown). Furthermore, when adding 'initial stroke severity' as a covariate in the statistical analyses, the association between $\triangle \mathrm{IGF}-\mathrm{I}$ and favorable 3-month outcome remained, whereas the association between $\triangle \mathrm{IGF}-\mathrm{I}$ and favorable 2-year outcome was weakened to statistically non-significant levels. Thus, even after correction for initial stroke severity, the association between $\triangle \mathrm{IGF}-\mathrm{I}$ and favorable 3-month functional outcome persisted.

In the multivariate regression analyses, the association between $\triangle \mathrm{IGF}-\mathrm{I}$ and IS outcome was not confounded by the level of acute s-IGF-I. This suggests that the association between $\triangle$ IGF-I and favorable outcome is independent of the acute level of IGF-I. Given the previous report on the absolute levels of IGF-I [5], we did not explore further the effects of the acute or 3-month levels of IGF-I.

The beneficial effect of $\Delta \mathrm{IGF}-\mathrm{I}$ was essentially unchanged by the applied adjustments for cardiovascular covariates (Fig. 3b and c). This is of importance, as the absolute levels of s-IGF-I are lower in cases of metabolic syndrome and diabetes [17-19]. In summary, the change in s-IGF-I level appears to be an independent predictor of favorable outcome 3 months after IS, as this association withstands corrections for cardiovascular covariates, absolute levels of acute IGF-I, and initial stroke severity.

\section{Possible underlying mechanisms}

In the present study, it was not possible to determine whether the changes in s-IGF-I reflect similar changes in the local availability of IGF-I in the brain. However, in experimental IS, IGF-I was upregulated early after IS, both locally $[7,8,20]$ and in the serum [8], allowing for a subsequent decrease in the level of IGF-I. In our previous study, the levels of s-IGF-I increased during the first days after the stroke. The levels of s-IGF-I reached a plateau on Days $3-5$, and thereafter, an average decrease of $14.8 \%$ was seen on Days 9-19, resulting in 3-month s-IGF-I levels that were approximately similar to those in healthy, age-matched controls [5]. It can be speculated that the higher levels of IGF-I around the brain injuries result in better recovery, and that after 3 months, the expression of IGF-I decreases if the injury exhibits recovery (implying that IGF-I is no longer needed). In line with this, brain injuries with little recovery would continue to have unchanged or even increased levels of IGF-I in the brain, and possibly also in the serum, after 3 months. If 
so, local IGF-I in the brain would not be an exacerbating agent, but instead a substance that could improve the clinical outcome of IS. From experimental studies, there is evidence that local astrocyte IGF-I expression mediates neuroprotection [21] and that locally delivered astrocyte IGF-I improves experimental stroke outcomes [22]. While the exact mechanisms are not known, they probably involve neuroprotection, as well as angiogenesis, neurogenesis, and neuronal sprouting (for reviews, see [1, 2]). Another possible explanation is that the patients with IS who exhibit a substantial recovery and favorable outcome are those with the greatest potential for transporting IGF-I from the serum into the brain [9]. Such a mechanism would give results similar to ours, although this notion is partly contradicted by our previous report that large infarctions have relatively higher absolute levels of both acute and 3-month s-IGF-I [5]. It should be pointed out that interactions between local brain IGF-I synthesis, circulating s-IGF-I (including peripheral sources and regulation from liver and bone [23]), and uptake of s-IGF-I through the blood-brain barrier are biologically plausible but very complicated to study and poorly understood in humans. A more stringent time series of IGF-I measurements in the serum and cerebrospinal fluid (CSF) in relation to IS might give some indication of the relative importance of the different sources of IGF-I.

\section{Changes in s-IGF-I levels in relation to previous studies and significance of favorable clinical outcome}

Our main finding is that $\triangle \mathrm{IGF}-\mathrm{I}$ is associated with favorable outcome both 3 months and 2 years post-IS, although the association with functional outcome after 2 years loses significance after adjustment for initial stroke severity. In a previous smaller study $(N=15)$, a decrease in IGF-I level during the first week of stroke was associated with shorter length of stay, greater independence at 1 month (mRS), and discharging to home vs. remaining as an inpatient [6]. As compared to the study of Mattlage and coworkers, our study has a wider range of first days of sampling and a considerably later time-point for the second sample (3 months). While the variation of the first day of sampling is a weakness in the present study, we have partly addressed this problem by correcting for this parameter in the multivariate regression analysis (Fig. 3, c and d, model 4) and by excluding Days 0-2, resulting in somewhat stronger associations between $\triangle$ IGF-I and favorable functional outcome (mRS $0-2$ ). Thus, the combined results of the present and the previous studies clearly suggest that a post-stroke decrease in the level of s-IGF-I is associated with improved clinical outcome after IS.

In the present study, the largest beneficial effects (ORs) observed for $\triangle \mathrm{IGF}$-I were in $\triangle \mathrm{IGF}-\mathrm{I}$-q3 (for 3-month outcomes) and in $\triangle \mathrm{IGF}-\mathrm{I}-\mathrm{q} 5$ (for 2-year outcomes), which correspond to decreases in s-IGF-I of 13.5 and $35.7 \%$, respectively (Table 1). These relatively substantial changes in s-IGF-I support the notion that s-IGF-I plays a role in stroke pathophysiology and rehabilitation. In terms of the crude unadjusted data, $42.3 \%$ of the patients who showed an increase in s-IGF-I ( $\triangle$ IGF-I-q1) had an unfavorable 3-month functional outcome, as compared to $12.7-20.3 \%$ of those patients who showed a decrease in s-IGF-I (q3 q5 of $\triangle \mathrm{IGF}-\mathrm{I})$. These are rather large differences, as an unfavorable outcome (mRS 3-6) means that the patient is requires assistance with daily living activities. Furthermore, the differences in associations noted between $\mathrm{q} 3$ and $\mathrm{q} 5$ for $\triangle \mathrm{IGF}-\mathrm{I}$ - and $\mathrm{mRS} 0-2$ are overall rather small. Our most important finding is that decreases in s-IGF-I levels contrast with no change or an increase in the post-stroke level of s-IGF-I with respect to outcome. However, this needs to be evaluated in greater detail in larger clinical studies.

\section{Strengths and limitations}

The methodological strengths of this study include consecutive recruitment of well-characterized patients with IS. Another advantage is the high hospitalization rate (84-95\%) for stroke patients in Sweden [24], which has among the highest rates in Europe [25]. The relatively young age of the participants in the present study (mean age, 55 years), as compared to the mean age of all patients with IS in Sweden (approximately 76 years [26]), facilitated follow-up, with very few drop-outs and few cases of fatality, although it somewhat disfavored the inclusion of the bulk of cases of IS etiology, i.e., IS due to cardiovascular causes. In addition, the inclusion of patients of young age favored the inclusion of less-severe cases of IS. The fact that few patients were lost to follow-up makes other selection biases unlikely. The fact that the patients were recruited between 1998 and 2003 means that very few patients received thrombolysis (local arterial, $N=4$, intravenous, $N=0)$ and that more of the patients received previous treatment with warfarin $(N=41)$ than is currently the case. Another drawback of this study is that there is no analysis of exact stroke volumes, although baseline stroke severity can be used as a marker of stroke lesion volumes with correlation coefficients of $0.62-0.64$ [27, 28]. In addition, we chose to include only those patients for whom there was a complete dataset for both the subacute and 3-month s-IGF-I, giving 354 subjects, as compared to the 407 subjects in our previous report [5]. We do not believe that this introduced any systematic bias, given that the cardiovascular covariates, acute and 3-month s-IGF-I levels, and the 3-month and 2-year mRS scores were comparable to those previously reported [5] (see also the Additional file 2: Table S1). Other weaknesses include the relatively small sample size and the lack of replication in a different geographic area. 


\section{Conclusions}

Decreasing levels of s-IGF-I show clear associations with favorable outcome at 3 months and 2 years after IS, suggesting that the dynamics of IGF-I regulation is of importance, independent of the actual s-IGF-I levels. After adjustment for initial stroke severity, the 3-month association remained statistically significant, whereas the 2-year association lost significance. Thus, the changes in s-IGF-I levels are associated primarily with the temporally close (3-month) outcomes, while the associations with long-term (2-year) outcomes are weakened and attenuated by other factors. The post-stroke changes in the levels of s-IGF-I are compatible with a positive role for IGF-I in IS recovery, although the exact mechanisms are uncertain and probably reflect certain combinations of different factors. Exploration of the causality warrants further studies involving intra-individual serial analyses of IGF-I levels in the serum and CSF of patients who have suffered an IS.

\section{Additional files}

Additional file 1: Supporting information. (DOCX $44 \mathrm{~kb}$ )

Additional file 2: Table S1. Baseline data as compared to the study in 2011 [5] (DOCX 33 kb)

\section{Abbreviations}

CI: 95\% confidence interval; IGF-I: Insulin-like growth factor I; IS: Ischemic stroke; mRS: Modified Rankin Scale; NIHSS: National Institutes of Health Stroke Scale; OCSP: Oxfordshire Community Stroke Project; SD: Standard deviation; SSS: Scandinavian Stroke Scale; TOAST: Trial of Org 10,172 in Acute Stroke Treatment

\section{Acknowledgments}

The authors wish to thank Dr. Vincent Collins for careful correction of the English language of the manuscript.

\section{Funding}

This study was supported by grants from the Swedish Government (ALFGBG751111, ALFGBG-719761, the Swedish Research Council (K2015-63X-2011710-4), the Swedish Heart Lung Foundation (20100256), the Swedish Stroke Association, the Göteborg Foundation for Neurological Research, and the Yngve Land, Rune and Ulla Amlöv, Edit Jacobson, Magnus Bergvall, Emelle, Lars Hierta, and John and Brit Wennerström foundations.

\section{Availability of data and materials}

The datasets used and/or analyzed during the current study are available from the corresponding author upon reasonable request.

\section{Authors' contributions}

NDÅ planned, designed, performed, analyzed, and wrote the paper. DA, MN, $\mathrm{CB}$ and HGK planned and wrote the paper. KJ planned, analyzed and wrote the paper. JS wrote the paper. JI and CJ planned, designed and wrote the paper. All authors contributed to and have approved the final manuscript.

\section{Ethics approval and consent to participate}

This study was conducted in accordance with the 1964 Helsinki Declaration and its later amendments or comparable ethical standards. Participants or ext-of-kin provided written informed consent. Specifically, next-of-kin consent was obtained if the participant was unable to communicate verbally or by writing, in accordance with the original criteria in the ethics application. This study was approved by the Ethics Committee of the University of Gothenburg. The Ethics Committee of the University of
Gothenburg is responsible for ethical applications from the entire region of western Sweden, covering all four hospitals in the study.

\section{Consent for publication}

Not applicable.

\section{Competing interests}

The authors declare that they have no competing interests.

\section{Publisher's Note}

Springer Nature remains neutral with regard to jurisdictional claims in published maps and institutional affiliations.

\section{Author details}

${ }^{1}$ Department of Internal Medicine, Institute of Medicine, The Sahlgrenska Academy at University of Gothenburg, Gröna Stråket 8, SE-413 45 Gothenburg, Sweden. ${ }^{2}$ Department of Clinical Neuroscience, Institute of Neuroscience and Physiology, The Sahlgrenska Academy at University of Gothenburg, Gothenburg, Sweden. ${ }^{3}$ Hunter Medical Research Institute, University of Newcastle, Newcastle, Australia. ${ }^{4}$ Center for Stroke Research Berlin, Charité - Universitätsmedizin Berlin, Berlin, Germany. ${ }^{5}$ Institute of Biomedicine, The Sahlgrenska Academy at University of Gothenburg, Gothenburg, Sweden. ${ }^{6}$ Department of Clinical Genetics, The Sahlgrenska Academy at University of Gothenburg, Gothenburg, Sweden.

Received: 8 March 2018 Accepted: 22 July 2018

Published online: 06 August 2018

References

1. Åberg ND, Brywe KG, Isgaard J. Aspects of growth hormone and insulin-like growth factor-I related to neuroprotection, regeneration, and functional plasticity in the adult brain. TheScientificWorldJournal. 2006;6:53-80.

2. Sohrabji F, Williams M. Stroke neuroprotection: oestrogen and insulin-like growth factor- 1 interactions and the role of microglia. J Neuroendocrinol. 2013:25(11):1173-81.

3. Denti L, Annoni V, Cattadori E, Salvagnini MA, Visioli S, Merli MF, Corradi F, Ceresini G, Valenti G, Hoffman AR, et al. Insulin-like growth factor 1 as a predictor of ischemic stroke outcome in the elderly. Am J Med. 2004;117(5): 312-7.

4. Bondanelli M, Ambrosio MR, Onofri A, Bergonzoni A, Lavezzi S, Zatelli MC, Valle D, Basaglia N, Degli Uberti EC. Predictive value of circulating insulinlike growth factor I levels in ischemic stroke outcome. J Clin Endocrinol Metab. 2006;91(10):3928-34.

5. Åberg D, Jood K, Blomstrand C, Jern C, Nilsson M, Isgaard J, Åberg ND. Serum IGF-I levels correlate to improvement of functional outcome after ischemic stroke. J Clin Endocrinol Metab. 2011;96(7):E1055-64.

6. Mattlage AE, Rippee MA, Sandt J, Billinger SA. Decrease in insulin-like growth Factor-1 and insulin-like growth Factor-1 ratio in the first week of stroke is related to positive outcomes. J Stroke Cerebrovasc Dis. 2016;25(7): 1800-6.

7. Beilharz EJ, Russo VC, Butler G, Baker NL, Connor B, Sirimanne ES, Dragunow M, Werther GA, Gluckman PD, Williams CE, et al. Co-ordinated and cellular specific induction of the components of the IGF/IGFBP axis in the rat brain following hypoxic-ischemic injury. Brain Res Mol Brain Res. 1998;59(2):119-34.

8. Wang J, Tang Y, Zhang W, Zhao H, Wang R, Yan Y, Xu L, Li P. Insulin-like growth factor-1 secreted by brain microvascular endothelial cells attenuates neuron injury upon ischemia. FEBS J. 2013;280(15):3658-68.

9. Carro E, Nunez A, Busiguina S, Torres-Aleman I. Circulating insulin-like growth factor I mediates effects of exercise on the brain. J Neurosci. 2000;20(8):2926-33.

10. Jood K, Ladenvall C, Rosengren A, Blomstrand C, Jern C: Family history in ischemic stroke before 70 years of age: the Sahlgrenska Academy study on ischemic stroke. Stroke 2005, 36(7):1383-1387.

11. Ali K, Cheek E, Sills S, Crome P, Roffe C. Development of a conversion factor to facilitate comparison of National Institute of Health Stroke Scale scores with Scandinavian Stroke Scale scores. Cerebrovasc Dis. 2007:24(6):509-15.

12. Bamford J, Sandercock P, Dennis M, Burn J, Warlow C. Classification and natural history of clinically identifiable subtypes of cerebral infarction. Lancet. 1991;337(8756):1521-6.

13. Adams HP Jr, Bendixen BH, Kappelle LJ, Biller J, Love BB, Gordon DL, Marsh EE 3rd. Classification of subtype of acute ischemic stroke. Definitions for use 
in a multicenter clinical trial. TOAST. Trial of org 10172 in acute stroke treatment. Stroke. 1993;24(1):35-41.

14. Banks JL, Marotta CA. Outcomes validity and reliability of the modified Rankin scale: implications for stroke clinical trials: a literature review and synthesis. Stroke. 2007;38(3):1091-6.

15. Åberg ND, Åberg D, Jood K, Nilsson M, Blomstrand C, Kuhn HG, Svensson J, Jern C, Isgaard J. The change in circulating insulin-like growth factor I (IGF-I) after ischemic stroke is independently associated with outcome. In: European Stroke Organisation Conference: 2018. Gothenburg: European Stroke Journal; 2018. p. 342-3. AS316-018.

16. Peduzzi P, Concato J, Kemper E, Holford TR, Feinstein AR. A simulation study of the number of events per variable in logistic regression analysis. J Clin Epidemiol. 1996;49(12):1373-9.

17. Landin-Wilhelmsen K, Wilhelmsen L, Lappas G, Rosen T, Lindstedt G, Lundberg PA, Bengtsson BA. Serum insulin-like growth factor I in a random population sample of men and women: relation to age, sex, smoking habits, coffee consumption and physical activity, blood pressure and concentrations of plasma lipids, fibrinogen, parathyroid hormone and osteocalcin. Clin Endocrinol. 1994;41(3):351-7.

18. Parekh N, Roberts CB, Vadiveloo M, Puvananayagam T, Albu JB, Lu-Yao GL. Lifestyle, anthropometric, and obesity-related physiologic determinants of insulin-like growth factor-1 in the third National Health and nutrition examination survey (1988-1994). Ann Epidemiol. 2010;20(3):182-93.

19. Spartano NL, Stevenson MD, Xanthakis V, Larson MG, Andersson C, Murabito $J M$, Vasan RS. Associations of objective physical activity with insulin sensitivity and circulating adipokine profile: the Framingham heart study. Clinical obesity. 2017;7(2):59-69.

20. Gustafson K, Hagberg H, Bengtsson BÅ, Brantsing C, Isgaard J. Possible protective role of growth hormone in hypoxia-ischemia in neonatal rats. Pediatr Res. 1999;45(3):318-23.

21. Genis L, Davila D, Fernandez S, Pozo-Rodrigalvarez A, Martinez-Murillo R Torres-Aleman I. Astrocytes require insulin-like growth factor I to protect neurons against oxidative injury. F1000Research. 2014;3:28.

22. Okoreeh AK, Bake S, Sohrabji F. Astrocyte-specific insulin-like growth factor1 gene transfer in aging female rats improves stroke outcomes. Glia. 2017; 65(7):1043-58.

23. Sjögren K, Liu JL, Blad K, Skrtic S, Vidal O, Wallenius V, LeRoith D, Törnell J, Isaksson OG, Jansson JO, et al. Liver-derived insulin-like growth factor I (IGFI) is the principal source of IGF-I in blood but is not required for postnatal body growth in mice. Proc Natl Acad Sci U S A. 1999;96(12):7088-92.

24. Hallström B, Jonsson AC, Nerbrand C, Petersen B, Norrving B, Lindgren A. Lund stroke register: hospitalization pattern and yield of different screening methods for first-ever stroke. Acta Neurol Scand. 2007;115(1):49-54.

25. Malmivaara $A$, Meretoja $A$, Peltola $M$, Numerato $D$, Heijink $R$, Engelfriet $P$, Wild SH, Belicza E, Bereczki D, Medin E, et al. Comparing ischaemic stroke in six European countries. The EuroHOPE register study. Eur J Neurol. 2015; 22(2):284-91. e225-286

26. Asplund K, Hulter Asberg K, Appelros P, Bjarne D, Eriksson M, Johansson A Jonsson F, Norrving B, Stegmayr B, Terent A, et al. The Riks-stroke story: building a sustainable national register for quality assessment of stroke care. Int J Stroke. 2011;6(2):99-108.

27. Effect of intravenous recombinant tissue plasminogen activator on ischemic stroke lesion size measured by computed tomography. NINDS; the National Institute of Neurological Disorders and Stroke (NINDS) rt-PA stroke study group. Stroke 2000, 31(12):2912-2919.

28. Menezes NM, Ay H, Wang Zhu M, Lopez CJ, Singhal AB, Karonen JO, Aronen HJ, Liu Y, Nuutinen J, Koroshetz WJ, et al. The real estate factor: quantifying the impact of infarct location on stroke severity. Stroke. 2007;38(1):194-7.

Ready to submit your research? Choose BMC and benefit from:
- fast, convenient online submission
- thorough peer review by experienced researchers in your field
- rapid publication on acceptance
- support for research data, including large and complex data types
- gold Open Access which fosters wider collaboration and increased citations
- maximum visibility for your research: over 100M website views per year
At BMC, research is always in progress.
Learn more biomedcentral.com/submissions

